\section{ENZYMES AND METABOLISM}

\section{Advances in Enzymology and Related Subjects of Biochemistry}

Vol. 21. Edited by F. F. Nord. Pp. v+521. (New York: Interscience Publishers, Inc.; London : Interscience Publishers, Ltd., 1959.) 12.50 dollars.

rTHIS volume of "Advances in Enzymology" con tains a very important set of articles on some important lines of metabolism which have only recently been elucidated. The synthesis of purines and pyrimidines is covered by three reviews which supplement each other : "Enzymic Reactions in the Synthesis of Purines", by Drs. Buchanan and Hartman ; "The Enzymic Synthesis of Pyrimidines", by Dr. Reichard; and "Folic Acid Coenzymes and One-Carbon Metabolism", by Drs. Huennekens and Osborn. In the past ten years the main lines of synthesis have been cleared up, with an appreciable increase in the number of known enzymes; some new and interesting enzymatic mechanisms are involved, and these are discussed critically in the first-mentioned article.

Other metabolic topies dealt with in the volume are oxidation and phosphorylation (in two reviews, by Dr. W. C. Schneider and Dr. D. E. Green, respectively) and the biosynthesis of carotenoid pigments (by Dr. T. W. Goodwin). Schneider's article reviews work on mitochondrial metabolism up to about 1957. Green's article is primarily concerned with the work of his own school on electron transport, which is discussed in terms of the special terminology which they have developed to express their view of the structural organization of enzymes in mitochondria. The enzymology of carotenoid metabolism is not as yet far developed, and Goodwin's contribution is necessarily mainly concerned with the chemistry of these plant products.

Two articles are concerned with the role of metals in enzymatic systems. Drs. Malmström and Rosenberg discuss (in English) the mechanism of metal ion activation of enzymes; Prof. Bamann and Dr. Trapmann are concerned (in the only article in German in this volum $\theta$ ) with reactions in which metal ion catalysis may be regarded as a model of the enzymatic catalysis. They give an excellent picture of the field, but leave the impression that much remains to be done.

This volume will clearly remain for some time to come a standard reference for the topics discussed therein.

EDWIN C. WeBb

\section{CONDUCTION IN SOLIDS}

\section{Electrons and Phonons}

The Theory of Transport Phenomena in Solids. By Dr. J. M. Ziman. (The International Series of Monographs on Physies.) Pp. xiv +554. (Oxford: Clarendon Press; London: Oxford University Press, 1960.) 84s. net.

L

ITTLE more than four years elapsed after the 1 discovery of wave mechanics before the basic principles of electrical and thermal conduction in metals were established, and such was the speed of the advance that within another four years, up to the end of 1934, the properties of individual metals, and even alloys, were being successfully analysed in terms of the new theory. The War brought progress in this field almost to a standstill, but at its end a new generation of physicists with new techniques and new ideas took up the pursuit once more, and the past fifteen years have seen a vast accumulation of detailed information on the properties of solids of many kinds. Although during this time there has been little change in the underlying principles or even in the mathematical methods employed in the electron theory of solids, yet so great a refinement of treatment has been introduced and so many more experimental effects discovered that, as the author of the book under review states in his preface, it is now probably beyond the capacity of any one man to write alone a satisfactory account of the whole theory.

In his book Dr. Ziman has confined himself to an account of the transport properties of metals, semiconductors and insulators; a subject of which he is undoubtedly a master. The title of the book at once indicates the quantal aspects of the conduction processes. By analogy with the quantized electromagnetic field it has become fashionable in recent years to refer to quantized elastic waves in crystals as phonons, and the whole mechanism of conduction is visualized as the mutual interaction between the phonons themselves and between phonons and electrons. This is an attractive picture, but it can all too easily suggest an over-simplification to the incautious reader: unlike a photon, the nature of a phonon in an elastically anisotropic crystal depends on the direction of its motion. The scattering of an elastic wave in a crystal is a more complicated process than that suggested by the deflexion of a single entity described as a phonon.

In an interesting analogy the author likens the writing of a scientific text-book to the process of purification by recrystallization. The imperfections of original papers are filtered away and the precipitate forms as a crystal in which each atom seems inevit. ably to be in its right place. This is a flattering description, and many of those with experience of either reading or writing text-books would hesitate to compare them with the perfect crystal. Dr. Ziman's is an excellent book but it will no doubt share the common lot and in the fulness of time will require extensive modification and revision. To the present generation of students-of all classes-the book will surely prove invaluable. It develops a theory in a clear and logical manner; dealing first with the properties of phonons, then with the properties of electrons in a periodic field, and eventually with the interactions between these systems. In this way is unfolded the theory of thermal conduction in insulators, the theory of thermal and electrical con. duction in metals, the effect of an external mag. netic field on the resistivity, the de Haas-van Alphen and the anomalous skin effects, and much besides.

The mathematics of the book is kept within reason. able bounds although always sufficiently detailed to enable the diligent reader to follow the argument completely. Physical concepts are emphasized, and the book abounds with discussions of significant experiments. The style is graceful, and erudite quotations at chapter headings are provided for the diversion of the reader. The publishers, for their part, have done a good job : the type is clear and the diagrams beautifully reproduced. Altogether this is an excellent book and deserves to be widely read.

H. JoNES 